\title{
Freight Car Dispatching with Generalized Flows
}

\author{
B.Engels ${ }^{\mathrm{a}, *}$, R.Schrader ${ }^{\mathrm{a}}$ \\ ${ }^{a}$ Dept. of Computer Science, University of Cologne, Weyertal 80, 50931 Cologne, Germany
}

\begin{abstract}
In the freight car dispatching problem empty freight cars have to be assigned to known demands respecting a given time horizon and certain constraints. The goal is to minimize the resulting transportation costs. One of the constraints is that customers can specify the type of cars they want. It is possible, however, that cars of certain types can be substituted by other cars, either in a 1-to-1 fashion or at different exchange rates. We show that these substitutions make the dispatching problem hard to solve and hard to approximate. We model the dispatching problem as an integral generalized transportation problem on a bipartite graph. Using rounding techniques, the LP-relaxation can be transformed to a transportation schedule violating some of the constraints slightly. Under an additional assumption on the cost function we fix this violation and derive a 4-approximation of the problem.
\end{abstract}

Key words: transportation, logistics, dispatching, generalized flow, complexity, approximation, heuristic

\section{Introduction}

The general concern of a cargo railway company is to transport goods between different customer sites. For this, empty freight cars have to be brought to the initial location to get loaded and have to be collected at their destination after unloading. The transportation of different goods imposes requirements on the freight cars (e.g. open or closed, bulk cargo or coil transport). Cars are therefore distinguished into different car types. It is possible, however, that a transportation demand may be served by cars of different types, as specified by allowed substitutions. These substitutions may come in a 1-to-1 fashion or at different 'exchange rates'. For some bulk cargo, for example, it may be that three cars of type A have the same capacity as five cars of type B. So the rules may allow to replace every five cars of type B by three cars of type A (and vice versa).

If the freight cars are owned by the railway company, apart from the transport of loaded freight cars, the company also has to manage the rental of its

${ }^{*}$ Corresponding author 
freight car stock. In the established work flow, the allocation of empty freight cars is treated as a separate dispatching problem: assign the available empty freight cars to given customer demands (or storages) with minimal total transport costs. Besides various (technical or marketing-oriented) side constraints, a valid dispatching has to respect a given freight train schedule and a set of substitution rules.

\subsection{Previous work}

There is a large number of optimization problems occurring in the context of the railroad industry. Strategic, tactical and operational topics are addressed in the literature beginning in the 1960s (see, e.g., the survey by Newman [16]). Within the context of the operational dispatching problem, other mid and short termed tasks emerge such as timetabling, blocking (cf., e.g., [3]) and scheduling problems (cf., e.g. [6, 7]). These problems are often modeled as certain network flow problems or network design problems. A detailed review with respect to such network models is given in [2]

One of the first approaches in literature to the distribution of empty freight cars as an optimization problem was a time-space expanded network model by White and Bomberault [25]. According to Gorman et al. [9, 10], CSX, one of the major US railway companies, used a decision-support tool developed by Turnquist and Markowicz [24] for equipment distribution until 1996 and later implemented the first real-time, fully integrated optimization system (DCP). On an operational level as well as on a tactical level similar systems have been implemented for the BNSF Railway [9], the Union Pacific Railway [15], the Swedish Railways $[11,13])$ and Canadian Pacific Railway [12]. Stochastic aspects of the problem were introduced by Powell and Carvalho $[18,19]$ and later combined with a multi-commodity flow model by Topaloglu and Powell [22]. The latter provides the basis for a (heuristic) decision support system for locomotive scheduling implemented at Norfolk Southern Railroad [10, 16]. A survey of dynamic and stochastic models for the allocation of empty containers is given by Crainic et al. [8].

Most of these approaches are based on flow models or multicommodity flow models. In the latter case, integrality requirements lead to mixed integer program formulations which are solved heuristically or with the help of LP-solvers, branch \& bound \& cut, decomposition and relaxation approaches. They do not consider, however, the substitution of cars. In the context of stock planning, the influence of 1-to-1 substitution rules is discussed, e.g., in Pentico [17].

\subsection{Our contribution}

We consider the dispatching of freight cars where substitutions between car types of arbitrary exchange rates are allowed. While these substitutions lead to more flexibility in the planning process, we show that they also make the problem hard to solve in a theoretical sense. We introduce a generalized transportation problem as a model for the dispatching problem and prove that even a restricted version of the problem cannot be approximated, unless $\mathcal{P}=\mathcal{N} \mathcal{P}$. 
Under further assumptions, based on investigations of the fractionality of its LP-solutions, we describe a rounding and rerouting heuristic and show that it yields a 4-approximation.

\subsection{Outline and some notation}

In Section 2 we introduce the generalized transportation problem, discuss its complexity status and the fractionality of its LP-relaxation. We explain the freight car dispatching problem in more detail in Section 3 and show how it fits into the framework of integral generalized transportation problems. We argue that we obtain a fractional dispatching of bounded fractionality by transforming the associated generalized network to a classical network. Finally, Section 4 presents two approximation algorithms based on these fractional solutions.

We use standard notations from graph theory. If not mentioned otherwise, all graphs we consider are undirected. If $e=(u, v)$ is an edge, $u$ is a neighbor of $v$ and $v$ is a neighbor of $u$. For a vertex $v \in V, \delta(v)$ denotes the set of all neighbors of $v$ and $\operatorname{deg}(v)=|\delta(v)|$ its degree. For $S \subseteq V, \delta(S)$ is the set of all vertices in $V \backslash S$ having at least one neighbor in $S$.

The notions we use from complexity theory can be found in, e.g., [4]. Let $\rho \geq 1$, and let $x$ be a feasible solution for a minimization problem. We call $x$ a $\rho$-approximation if its objective function value is at most $\rho$ times the optimal objective function value. The minimization problem is in the class $\mathcal{A} \mathcal{P} \mathcal{X}$ if for some $\rho \geq 1$ there is a polynomial algorithm producing a $\rho$-approximation for all instances of the problem.

\section{Generalized Transportation}

Let $G=(S \cup T, E)$ be a bipartite graph with the following three functions on its edges and vertices, respectively: a cost function $c: E \rightarrow \mathbb{R}$, a multiplier function $m: E \rightarrow \mathbb{R}$, and a balance function $b: S \cup T \rightarrow \mathbb{R}_{+}$. A generalized transportation is a vector $x \in \mathbb{R}_{+}^{E}$ such that

$$
\begin{array}{rlrl}
\sum_{e \in \delta(v)} x(e) & =b(v) & & \text { for all } v \in S \\
\sum_{e \in \delta(v)} m(e) x(e) & \leq b(v) & \text { for all } v \in T
\end{array}
$$

Interpreting $S$ as a set of supply vertices and $T$ as a set of demand vertices, a generalized transportation is a feasible solution of a transportation problem where the amounts on the receiving side are scaled by gains or losses along the edges. The cost $c(x)=\sum_{e \in E} c(e) x(e)$ of a generalized transportation is the same as in the usual transportation problem. An integral generalized transportation is a generalized transportation $x$ with $x \in \mathbb{N}$. 


\subsection{A reduction from a SAT-problem}

By a standard construction, for given $(G, m, b, c)$ the computation of a minimum cost generalized transportation can be modeled as a min-cost generalized flow problem over a bipartite directed graph with one additional sink and source (cf., e.g., [1]). In the continuous case, min-cost generalized flows can be computed in polynomial time, even for arbitrary directed graphs (cf., e.g., Tardos and Wayne [21]). In the integral case, however, the problem is $\mathcal{N} \mathcal{P}$-complete for arbitrary digraphs (cf. Sahni [20]). Beygang et al. [5] observed that Sahni proved $\mathcal{N} \mathcal{P}$-completeness already for series-parallel digraphs. In the following we prove that even restricted versions of the min-cost integral generalized transportation problem are $\mathcal{N} \mathcal{P}$-complete. For this, we use a reduction from the following satisfiability problem, which was shown to be $\mathcal{N} \mathcal{P}$-complete in [23]:

[3V2L3SAT] Given a Boolean 3-SAT formula $\alpha$ in which each variable occurs at most three times and each literal occurs at most two times, decide whether $\alpha$ is satisfiable.

Let $\alpha=C_{1} \wedge \cdots \wedge C_{k}$ with $C_{i}=l_{i 1} \vee l_{i 2} \vee l_{i 3}$ and $l_{i t} \in\left\{y_{j}, \neg y_{j}: 1 \leq j \leq\right.$ $n\} \cup\{\emptyset\}$ be a 3 V2L3SAT-formula with $n$ variables in $k$ clauses. We construct a generalized transportation instance on a complete bipartite graph $G=(S \cup T, E)$ with $m: E \rightarrow\{1,2\}$ such that there exists an integral generalized transportation $x$ with cost $k+5 n$ if and only if $\alpha$ is satisfiable.

The set $S$ consists of four sets $S_{C}$ and $S^{1}, S^{2}, S^{3}$ corresponding to clauses and variables in $\alpha$. For each clause $C_{i}, S_{C}$ contains one vertex $s_{i}$ and for each variable $y_{j}, S$ contains three vertices $s_{j}^{1}, s_{j}^{2}, s_{j}^{3}$. The demand $T$ contains for each variable $y_{j}$ four vertices $t_{j}^{1}, t_{j}^{2}, \vec{t}_{j}, \bar{t}_{j}^{2}$, two for each of the literals. We set $b(v)=1$ for all $v \in S_{C} \cup S^{2}$ and $b(v)=2$ for $v \in T \cup S^{1} \cup S^{3}$. We set $m(e)=1$ for all edges $e \in \delta(S) \backslash \delta\left(S^{2}\right)$ and $m(e)=2$ for $e \in \delta\left(S^{2}\right)$. The cost of edges in the following list is set to 1 :

$$
\begin{array}{ll} 
& \left\{\left(s_{i}, t_{j}^{1}\right): C_{i} \text { is the first clause containing } y_{j}\right\} \\
\cup & \left\{\left(s_{i}, t_{j}^{2}\right): C_{i} \text { is the second clause containing } y_{j}\right\} \\
\cup & \left\{\left(s_{i}, \bar{t}_{j}^{1}\right): C_{i} \text { is the first clause containing } \neg y_{j}\right\} \\
\cup & \left\{\left(s_{i}, \bar{t}_{j}^{2}\right): C_{i} \text { is the second clause containing } \neg y_{j}\right\} \\
\cup & \left\{\left(s_{j}^{1}, t_{j}^{1}\right),\left(s_{j}^{1}, t_{j}^{2}\right): j=1, \ldots, n\right\} \\
\cup & \left\{\left(s_{j}^{2}, t_{j}^{2}\right),\left(s_{j}^{2}, \bar{t}_{j}^{1}\right): j=1, \ldots, n\right\} \\
\cup & \left\{\left(s_{j}^{3}, \bar{t}_{j}^{1}\right),\left(s_{j}^{3}, \bar{t}_{j}^{2}\right): j=1, \ldots, n\right\}
\end{array}
$$

All other edges have cost 2 .

Figure 1 illustrates the construction for variable $y_{j}$ occurring unnegated in clauses $C_{i}$ and $C_{k}$ and negated in clause $C_{\ell}$. The edges drawn have cost 1 , all other edges (having cost 2) are omitted. The multiplier on a bold edge is 2 , all other edges have multiplier 1 . The numbers at the vertices represent their $b$-value. 


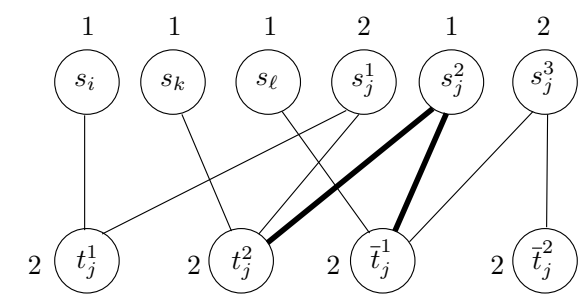

Figure 1: The gadget associated with variable $j$ occurring as $y_{j}$ in clauses $C_{i}$ and $C_{k}$ and as $\neg y_{j}$ in clause $C_{\ell}$.

Since the shipping cost per unit is at least one and since we have to ship one unit for each clause and five units for each variable, the total transportation cost is at least $k+5 n$.

Lemma 1. Let $x$ be an integral generalized transportation for the above problem with $c(x)=k+5 n$. Then for all $1 \leq j \leq n$ one of the following conditions holds:

(i) $x\left(s_{j}^{1}, t_{j}^{1}\right)=2, x\left(s_{j}^{2}, t_{j}^{2}\right)=1$ and (a) $x\left(s_{j}^{3}, \bar{t}_{j}^{1}\right)=2$ or (b) $x\left(s_{j}^{3}, \bar{t}_{j}^{2}\right)=2$ or (c) $x\left(s_{j}^{3}, \vec{t}_{j}^{1}\right)=x\left(s_{j}^{3}, \vec{t}_{j}^{2}\right)=1$

(ii) $x\left(s_{j}^{3}, \bar{t}_{j}^{2}\right)=2, x\left(s_{j}^{2}, \bar{t}_{j}^{1}\right)=1$ and (a) $x\left(s_{j}^{1}, t_{j}^{1}\right)=2$ or (b) $x\left(s_{j}^{1}, t_{j}^{2}\right)=2$ or (c) $x\left(s_{j}^{1}, t_{j}^{1}\right)=x\left(s_{j}^{1}, t_{j}^{2}\right)=1$

Proof: Let $x$ be an integral generalized transportation with $c(x)=k+5 n$. Since we have to ship $k+5 n$ units out of the vertices in $S$, they all have to be transported at cost 1 . The only way the one unit of supply at vertex $s_{j}^{2}$ can be transported at cost 1 is to ship it to either $t_{j}^{2}$ or to $\vec{t}_{j}^{1}$. In the first case, the demand of $t_{j}^{2}$ is satisfied so that the two units at $s_{j}^{1}$ have to be sent to $t_{j}^{1}$. Hence we are in case (i) and the subcases distinguish the three possibilities to ship the supply of $s_{j}^{3}$ at cost 1 . With similar arguments, the second case leads to (ii).

Observe that in case (i) the inequalities (2) for $t_{j}^{1}$ and $t_{j}^{2}$ hold with equality, while for $\vec{t}_{j}$ and $\vec{t}_{j}^{1}$ at least one has some slack. In case (ii) it is the other way around. We identify the three cases in (i) with $y_{j}=$ false and the cases in (ii) with $y_{j}=$ true.

Theorem 1. The formula $\alpha$ is satisfiable if and only if there is an integral generalized transportation $x$ in $(G, m, b, c)$ with cost $c(x)=k+5 n$.

Proof: Let $\alpha$ be satisfiable and $y$, a satisfying truth assignment. According to the truth values of $y$ and the rules of Lemma 1 we ship the supply of $S^{1} \cup S^{2}$ to $T$. This transportation costs $2\left|S^{1}\right|+\left|S^{2}\right|=3 n$. No matter what subrule we choose, the inequalities (1) will then hold for all $v \in S^{1} \cup S^{2}$. We now specify 
which of the subrules of (i) resp. (ii) we choose. Consider clause $C_{1}$. Since $y$ is a satisfying assignment, $C_{1}$ has a literal, $y_{j}$ say, with $y_{j}=$ true. Hence we are in case (ii). By definition, $y_{j}$ occurs in at most two clauses. If $y_{j}$ also occurs in some clause $C_{i}$, we apply subrule (c) and set $x\left(s_{1}, t_{j}^{1}\right)=x\left(s_{j}^{1}, t_{j}^{2}\right)=1$. If $C_{1}$ is the only clause containing $y_{j}$, then we apply subrule (b) and set $x\left(s_{1}, t_{j}^{1}\right)=2$. In both cases the inequalities (2) for $t_{j}^{1}, t_{j}^{2}, \vec{t}_{j}^{1}, \bar{t}_{j}^{2}$ are fulfilled and the equation (1) holds for $s_{j}^{3}, s_{1}$ (and in subcase (c) for $s_{i}$ ). Proceeding this way, we add another cost term of 2 for each variable and a cost term of one for each clause and obtain an integral generalized transportation $x$ with cost $c(x)=k+5 n$.

Conversely, let $x$ be an integral generalized transportation in $G=(S \cup T, E)$ with cost $c(x)=k+5 n$. By Lemma 1, $x$ satisfies the rules (i) and (ii), and induces a truth assignment $y$ as before. We claim that $y$ satisfies $\alpha$. Suppose not. Then there is a clause which is not satisfied. After some rearrangement let $C_{1}=y_{1} \vee y_{2} \vee y_{3}$ be this clause. There are only six edges in $\delta\left(s_{1}\right)$ with cost 1 , namely $\left(s_{1}, t_{i}^{1}\right)$ and $\left(s_{1}, t_{i}^{2}\right)$ for $i=1,2,3$. However, since case (i) applies, the demand of the vertices $\left(t_{i}^{1}\right)$ and $\left(t_{i}^{2}\right)$ for $i=1,2,3$ is already saturated by rule (i). Hence, the one unit of $s_{1}$ can only be shipped at cost 2 , contradicting the assumption.

\subsection{Complexity of a restricted version}

Observe that we have a certain structure in the generalized transportation problem used in the proof of Theorem 1. First, we have

$$
m(s, u)=m(s, v) \text { for all } s \in S \text { and all }(s, u),(s, v) \in E .
$$

In general, this property need not hold. It may hold, however, after some scaling. Multiplying the inequalities in (2) with $w(v)>0, v \in T$ leaves the feasible region unaffected. We call a generalized transportation problem homogeneous if (after scaling with some $w$ ) we have $w(u) m(s, u)=w(v) m(s, v)$ for all $s \in S$ and all $(s, u),(s, v) \in E$. We henceforth assume that a homogeneous problem has already been scaled so that (3) holds. Secondly, our reduction uses very restricted multiplier functions and balance functions. We call a problem even if $m: E \rightarrow\{1,2\}$, the balance function $b$ is integral and if $b(t)$ is even for all $t \in T$ which are incident to an edge $e$ with $m(e)=2$. Finally, the cost function fulfills the following quadrilateral inequality:

$$
c(u, x) \leq c(u, w)+c(v, w)+c(v, x) .
$$

So we consider the following restricted generalized transportation problem:

[RestIGT] Given an even and homogeneous generalized transportation problem with multiplier function and a $k \in \mathbb{N}$, is there an integral generalized transportation with cost at most $k$ ?

Corollary 1. RestIGT is not in $\mathcal{A P \mathcal { X }}$ and is $\mathcal{N} \mathcal{P}$-complete even if the cost function satisfies (4). 
Proof: The $\mathcal{N} \mathcal{P}$-completeness follows from Theorem 1 . If we replace the cost parameter 2 by some $M$, we can make the gap between optimal and nonoptimal solutions of the generalized transportation problem arbitrarily large. Hence there cannot exist an approximation algorithm with fixed approximation ratio (unless $\mathcal{P}=\mathcal{N} \mathcal{P}$ ).

\subsection{Fractionality of solutions}

If $m(e)=1$ for all edges $e \in E$, the integral generalized transportation problem is a classical flow problem. In this case, if the input values are integral, we may assume that an optimal solution is also integral. In the case of an arbitrary multiplier function and without an integrality requirement, it seems natural to ask how fractional an optimal solution can be. In the context of multicommodity flows, this question was raised and partially answered by, e.g., Karzanov [14].

Given a bipartite graph $G=(S \cup T, E)$ and a multiplier function $m$, the fractionality of $(G, m)$ is the least $\beta \in \mathbb{N}$ such that any generalized transportation problem with integral balance function $b$ and arbitrary cost function $c$ has a $1 / \beta$-fractional optimal solution. In the following, we investigate the fractionality of homogeneous instances.

Let $m$ be the multiplier function of a homogeneous instance. Then for all $u \in T m$ is of the form $m(s, u)=p_{s} / q_{s}$ for some $p_{s}, q_{s} \in \mathbb{N}$. Let $p$ be the least common multiple of the $\left\{p_{s}: s \in S\right\}$ and $q$ be the least common multiple of the $\left\{q_{s}: s \in S\right\}$.

Lemma 2. Let $(G, m, b, c)$ be a homogeneous instance of the generalized transportation problem with $b$ integral and $p, q$ as above. Then $(G, m)$ is pq-fractional.

Proof: Let $(G, m, b, c)$ be a homogeneous instance with balance function $b \in \mathbb{N}^{S \cup T}$ and cost function $c$. We define a new instance $\left(G, m^{\prime}, b^{\prime}, c^{\prime}\right)$ as follows: $b^{\prime}(s)=\frac{p_{s}}{q_{s}} q b(s)$ for $s \in S, b^{\prime}(t)=q b(t)$ for $t \in T, m^{\prime}(s, t)=1$ and $c^{\prime}(s, t)=$ $\frac{q_{s}}{p_{s} q} c(s, t)$ for all $(s, t) \in E$. Then $\left(G, m^{\prime}, b^{\prime}, c^{\prime}\right)$ is a transportation problem and

hence has an integral solution $x^{\prime}$. Let $x(s, t)=\frac{q_{s}}{p_{s} q} x^{\prime}(s, t)$. Then

$$
\begin{aligned}
\sum_{t \in T} x(s, t) & =\sum_{t \in T} \frac{q_{s}}{p_{s} q} x^{\prime}(s, t)=\frac{q_{s}}{p_{s} q} b^{\prime}(s)=b(s) \\
\sum_{s \in S} m(s, t) x(s, t) & =\sum_{s \in S} \frac{p_{s}}{q_{s}} \frac{q_{s}}{p_{s} q} x^{\prime}(s, t)=\sum_{s \in S} \frac{1}{q} x^{\prime}(s, t) \leq b(t)
\end{aligned}
$$

So (1) and (2) hold and $x$ is a $1 / p q$-fractional generalized transportation for $(G, m, b, c)$ with the same objective function value. Conversely, if $x$ is a generalized transportation for $(G, m, b, c)$, then $x^{\prime}(s, t)=\frac{p_{s} q}{q_{s}} x(s, t)$ is a feasible transportation for $\left(G, m^{\prime}, b^{\prime}, c^{\prime}\right)$ with the same objective function value. Hence, $x^{\prime}$ is optimal for $\left(G, m^{\prime}, b^{\prime}, c^{\prime}\right)$ if and only if $x$ is optimal for $(G, m, b, c)$ and the claim follows. 
Observe that we have transformed the generalized transportation problem into a classical transportation problem. As this is polynomially solvable, we can obtain $p q$-fractional solutions in polynomial time.

\section{Application: freight car dispatching}

Very often in cargo railway transportation, cars are owned by some company (sometimes the railway company itself) and rented away to customers. These customers may transport various types of goods which require different freight cars (e.g. open or closed, bulk cargo or coil transport). Hence the rental company offers a fleet of various car types. Since the customers are located at different places and need various types of different cars at different points in time, the car rental requires a dispatching where new demands for cars are matched with cars that have been returned.

A demand is represented by a location, the type and the number of cars and a time when they have to be available at latest. In our model, a demand is represented by a vertex $t \in T$ with demand $b(t)$ given by the number of cars requested. Similarly, supplies are given by a location, the type and the number of returned cars and their earliest availability. They define vertices in $S$ with supply $b(s)$. Moreover, we may have certain depot vertices in $S$ and $T$ where cars can be stored temporarily.

The transportation of empty cars from a supply $s$ to a demand vertex $t$ is done by attaching them to trains running on a given timetable. Based on this timetable and the availabilities at the vertices $s$ and $t$ we can decide whether cars in $s$ can reach $t$ in time. If yes, we also have a way to compute the transportation cost of a single car from $s$ to $t$ (usually based on the distance the car travels). If also the car types at $s$ and $t$ match, we introduce an edge $e=(s, t)$ with costs $c(e)$. If the types do not match or the time restrictions cannot be met, $s$ and $t$ are not linked by an edge.

So far, the dispatching problem can be modeled as a classical transportation problem. However, since a demand can be satisfied by cars of several types, the problem becomes more complicated. In its general form, substitutions are of the form $\left(n_{i}, n_{j}\right)$ specifying that $n_{i}$ cars of type $i$ may replace $n_{j}$ cars of type $j$. The substitution rules are modeled by the function $m$ by setting $m(i, j)=\frac{n_{j}}{n_{i}}$. This leads to a generalized transportation problem $(G, m, b, c)$ on the bipartite graph $G=(S \cup T, E)$ just defined.

As an illustration, consider two supplies $s_{1}, s_{2}$ and two demands $t_{1}, t_{2}$ at certain locations. We assume that the time restrictions are such that cars form both supply locations may reach both demand locations in time. $s_{1}$ has a supply of 9 cars of type A, $s_{2}$ has 7 cars of type B. Demand $t_{1}$ wants up to 8 cars of type $\mathrm{A}, t_{2}$ needs up to 17 cars of type $\mathrm{C}$. Type $\mathrm{C}$ may be substituted in a 1-to- 1 fashion by type B. Moreover, every 5 cars of type $\mathrm{C}$ may be replaced by 3 cars of type A. This leads to the bipartite graph in Figure 2. The weights on the edges represent their $m$-values, the numbers at the vertices give their $b$-values. 


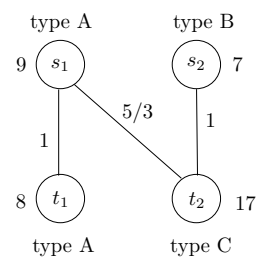

Figure 2: The bipartite graph associated with the example of the dispatching problem.

An integral generalized transportation is given by, e.g., $x\left(s_{1}, t_{1}\right)=3, x\left(s_{1}, t_{2}\right)=$ $6, x\left(s_{2}, t_{2}\right)=7$.

\subsection{Practical dispatching problems from DB Schenker Rail Deutschland AG}

In an R \& D project together with the optimization group of Sven O. Krumke (Technical University of Kaiserslautern) and DB Schenker Rail Deutschland AG we looked into real data from freight car dispatching problems arising at Schenker. We found that the dispatching rules used by Schenker satisfy most of the restrictions discussed in Section 2.2.

First, the cars they use not only have some type but also one of two lengths ("short" and "long"). The substitution rules only allow a simple 1-to-1 replacement between cars of the same length and of perhaps different types or a substitution of one long car of a certain type by two short cars of some other type. (The reverse substitution of two short cars by one long car could be rephrased using the previous rules.) Since this imposes substitution rules of the form $(2,1)$, it is not immediately obvious that the DB-instances are homogeneous. Observe, however, that a vertex $s \in S$ representing a long car can only serve a demand of long cars in $T$. If $s \in S$ represents a short car, it may 1-to-1 replace other short cars in $T$ (with $m(s, t)=1$ ) or 2-to-1 replace long cars (with $\left.m(s, t)=\frac{1}{2}\right)$. So these instances are seen to be homogeneous by setting $w(t)=2$ if $t \in T$ represents a long car and $w(t)=1$ for short cars. This scaling gives a new multiplier of 2 on edges $(s, t)$ between two vertices both representing long cars and of 1 on all other edges. Also note that after this scaling the problem is even.

Hence, we may apply Lemma 2 with $p=2$ and $q=1$. This allows us to compute half-integral generalized transportations $x(s, t)$ in polynomial time. More precisely, the construction in the proof of Lemma 2 shows that $x(s, t)$ is half-integral only if $s$ represents a long car and integral otherwise.

Secondly, transport costs are given by costs per kilometer. Hence the cost function $c$ trivially satisfies the quadrilateral inequality if whenever in (4) the edges $(u, w),(v, w),(v, x)$ exist also the edge $(u, x)$ exists, i.e. the supply at $u$ may serve the demand at $x$ and arrives there in time. (The latter condition was not always satysfied.) 


\section{Relaxed solutions for RestIGT}

In this section we assume that all instances of the generalized transportation problem are homogeneous and even. From Section 2 we know that we cannot expect to find polynomial time solutions with an approximation guarantee. We are going to show, however, that we can find solutions quickly if we allow some conditions to be violated slightly. In a second step we furthermore asssume that the quadrilateral inequality (4) holds. In this case, the problem remains $\mathcal{N} \mathcal{P}$-complete but we can find a 4 -approximation.

\subsection{A rounding heuristic}

For the first step we need a technical Lemma.

Lemma 3. Let $G=(S \cup T, E)$ be a bipartite graph with $\operatorname{deg}(v)$ even for all $v \in S$. Then $E$ can be partitioned into a set of even-length paths and and a set of even cycles.

Proof: Let $U=\left\{u_{1}, \ldots, u_{k}\right\}$ be the set of odd-degree vertices in $G$. Obviously, $k$ is even. Consider the graph $G=(S \cup T, E \cup F)$ where $F=$ $\left\{\left(u_{1}, u_{2}\right), \ldots,\left(u_{k-1}, u_{k}\right)\right\}$. Then $G$ is Eulerian and $E \cup F$ can be decomposed into cycles. Let $C$ be one of these cycles. If $C \cap F=\emptyset$ then $C$ is a cycle in $G$ and hence even. Otherwise, $C \backslash F$ decomposes into a set of paths, each starting and ending in vertices in $U$. Then also these paths must be even.

Since we assume that the generalized transportation problem $(G, m, b, c)$ is even, we have in particular that $m$ has values in $\{1,2\}$. By Lemma 2, we can find a half-integral optimal solution $x$ in polynomial time. Let $G^{\prime}=\left(S^{\prime} \cup T^{\prime}, E^{\prime}\right)$ be the subgraph of $G$ induced by the fractional entries of $x$, i.e.

$$
\begin{aligned}
E^{\prime} & =\{(s, t) \in E: x(s, t) \notin \mathbb{N}\} \\
S^{\prime} & =\left\{s \in S: x(s, t) \in E^{\prime} \text { for some } t \in T\right\} \\
T^{\prime} & =\left\{t \in T: x(s, t) \in E^{\prime} \text { for some } s \in S\right\} .
\end{aligned}
$$

As, by assumption, $x$ satisfies the equations (1), its fractional components are half-integral and $b(v) \in \mathbb{N}, \operatorname{deg}(v)$ must be even for $v \in S^{\prime}$. Hence, by Lemma $3, E^{\prime}$ can be partitioned into a set $\mathcal{P} \subseteq E^{\prime}$ of even-length paths and and a set $\mathcal{C} \subseteq E^{\prime}$ of even cycles.

Adopting notions from Section 3.1, we call a vertex "long" if it is incident to some edge $e$ with $m(e)=2$, and "short" otherwise. Recall from the constrution in Lemma 2 that every vertex in $S^{\prime} \cup T^{\prime}$ represents a long car and we have $m(s, t)=2$ for all $e \in E^{\prime}$.

While trying to keep the costs low, we now transform $x$ into an integral vector. This vector will still satisfy the equations (1) and violate the inequalities (2) slightly. For this, we round the half-integral entries of $x$ along edges of a 
path and of a cycle alternatingly up and down. Since $\mathcal{P}$ and $\mathcal{C}$ partition $E^{\prime}$, we thus obtain an integral vector $y$. Obviously, the rounding leaves vertices in $S^{\prime}$ unaffected. Hence the equations (1) still hold. Since $m(s, t)=2$ for all $e \in E^{\prime}$, also all vertices in $T^{\prime}$ which do not occur as end-vertices of paths are unaffected. The only vertices for which (2) may be violated are the end vertices of paths where the incoming path edge is rounded up. Let $v$ be such an end-vertex. Since $v \in U$, it is the endpoint of an odd number of half-integral edges. As $b(v)$ is even, before rounding up we either have $\sum_{e \in \delta(v)} m(e) x(e) \leq b(v)-1$ or $\sum_{e \in \delta(v)} m(e) x(e)=b(v)$ and $x(u, v) \geq 1$ for some $u \in S$ representing a short car. In the first case, $y$ still satisfies the corresponding inequality $(2)$, in the second case we have $\sum_{e \in \delta(v)} m(e) y(e) \leq b(v)+1$.

Consider a demand vertex $t \in T$ and two supply vertices $s_{1}, s_{2}$ with $\left(s_{1}, t\right)$, $\left(s_{2}, t\right) \in E$ and $m\left(s_{1}, t\right)<m\left(s_{2}, t\right)$. Neglecting for a moment the equations (1), we say that $t$ gets a downgrade if we replace one car of $s_{1}$ by one car of $s_{2}$ in an (integral) generalized transportation. For a given $\nu \geq 0$, we call a vector $x \in \mathbb{N}^{E}$ a $\nu$-relaxed generalized transportation if it satisfies the equations (1) and

$$
\sum_{e \in \delta(v)} m(e) x(e) \leq b(v)+\nu \text { for all } v \in T
$$

and after downgrading a single car for all $t \in T$ with $\sum_{e \in \delta(v)} m(e) x(e)>b(v)$ the inequalities (2) are satisfied.

Lemma 4. The rounded vector $y \in \mathbb{N}^{E}$ may be chosen such that it is a 1 relaxed generalized transportation with at most $\frac{1}{2}|U|$ over-satisfied demands and $\operatorname{cost} c(y) \leq c(x)$.

Proof: Given a path $P \in \mathcal{P}$ or a cycle $C \in \mathcal{C}$ with a specified orientation we still have the freedom to choose the direction of the first rounding. Let $c\left(P^{-}\right)$be the change of cost of rounding $x$ along $P$ by rounding the first edge down, and $c\left(P^{+}\right)$the change by rounding the first edge up. By flipping the orientation of $P$ if necessary, we may assume that $c\left(P^{+}\right) \leq c\left(P^{-}\right)$. Obviously, $2 c\left(P^{+}\right) \leq c\left(P^{+}\right)+c\left(P^{-}\right)=0$, i.e. $c\left(P^{+}\right) \leq 0$. A similar argument shows that we may assume $c\left(C^{+}\right) \leq 0$ for all $C \in \mathcal{C}$. Let $y \in \mathbb{N}^{E}$ be the result of this rounding strategy. Then $c(y) \leq c(x)$.

The discussion above shows that $\sum_{e \in \delta(v)} m(e) y(e) \leq b(v)+1$ and oversatisfaction can occur only in one of the end-vertices of a path, i.e. in at most $\frac{1}{2}|U|$ vertices. A vertex $t \in U$ is over-satisfied only if it has an excess of half a long car. Since $y$ is integral, $t$ must receive at least one long car. If we replace this long car by a short one, the corresponding inequality (2) is satisfied.

Note that from a practical point of view the dispatching $y$ may be attractive since it generates low costs and only for some customers $t \in T$ one of their short cars is replaced by one long car. This seems acceptable unless car storage capacity is a problem and the bound $b(t)$ is a hard constraint. 


\subsection{A 4-approximation}

We are now going to fix the violations of inequality (2). In the rounding process, instead of trying to keep the costs low, we may as well try to keep the number of over-satisfied demands low. So, if possible, we choose the rounding direction so that the vertex rounded up is not over-satisfied. Let $u$ be such an over-satisfied vertex. Let $v \in U$ be the other endpoint of the path along which we have rounded. Then, before rounding, we have $\sum_{e \in \delta(u)} m(e) x(e)=b(u)$ since otherwise we could reverse the path and decrease the number of oversatisfied vertices. By the remarks above, there is a vertex $w \in S$ corresponding to a short car with $x(w, u) \geq 1$. Since the problem is homogeneous, we can now redirect one short car from $w$ to $u$ and send it to $v$ instead. Then, by Lemma 4, the inequalities (2) are satisfied both in $u$ and $v$. Iterating this way, we get an integral generalized transportation $z$.

Theorem 2. Let $(G, m, b, c)$ be an instance from RestIGT satisfying the quadrilateral inequality (4). Then $z$ is a 4-approximation for $(G, m, b, c)$.

Proof: The transportation $z$ is obtained from the generalized transportation $x$ by rounding along paths in $\mathcal{P}$ and cycles in $\mathcal{C}$ and redirection of some cars. As observed before, the rounding along cycles causes no over-satisfaction, no matter what orientation we choose. So, if we choose the one with lower costs, rounding along cycles does not increase the costs.

Consider a path $P=v_{1}, v_{2}, \ldots, v_{2 k+1}$ and suppose we start with a roundup. Let $u \in S$ be the vertex from where one short car is redirected. Then the change $\Delta(P)$ in cost is

$$
\begin{aligned}
\Delta(P) & =-c\left(u, v_{1}\right)+\frac{1}{2} \sum_{\substack{i=1 \\
i \text { odd }}}^{2 k-1} c(i, i+1)-\frac{1}{2} \sum_{\substack{i=2 \\
i \text { even }}}^{2 k} c(i, i+1)+c\left(u, v_{2 k+1}\right) \\
& \leq-c\left(u, v_{1}\right)+\frac{1}{2} c(P)+c\left(u, v_{2 k+1}\right) \\
& \leq-c\left(u, v_{1}\right)+\frac{1}{2} c(P)+c\left(u, v_{1}\right)+c(P) \quad \text { (by (4)) } \\
& \leq \frac{3}{2} c(P) .
\end{aligned}
$$

Since $x(e) \geq \frac{1}{2}$ for each edge in $P$ and the paths in $\mathcal{P}$ are edge-disjoint, we get $c(z) \leq c(x)+\sum_{P \in \mathcal{P}} \Delta(P) \leq c(x)+\frac{3}{2} \sum_{P \in \mathcal{P}} c(P) \leq c(x)+3 c(x)=4 c(x)$.

Observe that the cost of $z$ is bounded in terms of the (non-integral) generalized transportation. The gap between optimal integral and non-integral generalized transportations may be be quite large so that the approximation ratio may be much smaller. 


\section{Acknowledgements}

The authors are grateful to three anonymous referees. Their questions, suggestions and comments greatly improved the presentation of the paper.

We also like to thank DB Schenker Rail Deutschland AG for sponsoring the $\mathrm{R} \& \mathrm{D}$ project and for providing the application data for our test instances and the optimization group of Sven O. Krumke from the Technical University of Kaiserslautern for the joint work on the project.

\section{References}

[1] R. K. Ahuja, T. L. Magnanti, and J. B. Orlin. Network flows: theory, algorithms, and applications. Prentice-Hall, Inc., Upper Saddle River, NJ, USA, 1993.

[2] R.K. Ahuja, C.B. Cunha, and G. Sahin. Network models in railroad planning and scheduling. Tutorials in Operations Research, 1:54-101, 2005.

[3] R.K. Ahuja, J. Liu, J.B. Orlin, D. Sharma, and L.A. Shughart. Solving reallife locomotive-scheduling problems. Transportation Science, 39:503-517, 2005.

[4] G. Ausiello, P. Crescenzi, G. Gambosi, V. Kann, A. Marchetti-Spaccamela, and M. Protasi. Complexity and Approximation. Springer-Verlag, Berlin, Heidelberg, New York, 2003.

[5] K. Beygang, S.O. Krumke, and C. Zeck. Generalized max flow in seriesparallel graphs, 2010. WIMA Report, Band 125.

[6] A. Caprara, M. Fischetti, and P. Toth. Modeling and solving the train timetabling problem. Operations Research, 50:851-861, 2002.

[7] A. Caprara, M. Monaci, P. Toth, and P.L. Guida. A lagrangian heuristic algorithm for a real-world train timetabling problem. Discrete Applied Mathematics, 154:738-753, 2006.

[8] T. G. Crainic, M. Gendreau, and P. Dejax. Dynamic and stochastic models for the allocation of empty containers. Operations Research, 41(1):102-126, 1993.

[9] M.F. Gorman, D. Acharya, and D. Sellers. CSX railway uses OR to cash in on optimized equipment distribution. Interfaces, 40(1):5-16, 2010.

[10] M.F. Gorman, K. Crook, and D. Sellers. North american freight rail industry real-time optimized equipment distribution systems: State of the practice. Transportation Research Part C: Emerging Technologies, 19(1):103114, 2011.

[11] K. Holmberg, M. Joborn, and J.T. Lundgren. Improved empty freight car distribution. Transportation Science, 32(2):163-173, 1998. 
[12] P. Ireland, R. Case, J. Fallis, C. Van Dyke, J. Kuehn, and M. Meketon. The Canadian Pacific Railway transforms operations by using models to develop its operating plans. Interfaces, 34(1):5-14, 2004.

[13] M. Joborn, T.G. Crainic, M. Gendreau, K. Holmberg, and J.T. Lundgren. Economies of scale in empty freight car distribution in scheduled railways. Transportation Science, 38:121-134, 2004.

[14] A. Karzanov. Multiflows and disjoint paths of minimum total cost. Mathematical Programming, 78:219-242, 1997.

[15] A.K. Narisetty, Richard J.-P. P., D. Ramcharan, D. Murphy, G. Minks, and J. Fuller. An optimization model for empty freight car assignment at Union Pacific Railroad. Interfaces, 38(2):89-102, 2008.

[16] A.M. Newman, L. Nozick, and C.A. Yano. Optimization in the Rail Industry, pages 704-718. Oxford University Press, New York (NY), 2002.

[17] D. Pentico. The assortment problem: a survey. European Journal of Operational Research, 190:295-309, 2008.

[18] W.B. Powell and T. Carvalho. Dynamic control of logistics queueing networks for large-scale fleet management. Transportation Science, 32(2):90109, 1998.

[19] W.B. Powell and T. Carvalho. Real-time optimization of containers and flatcars for intermodal operations. Transportation Science, 32(2):110-126, 1998.

[20] S. Sahni. Computationally related problems. SIAM Journal on Computing, $3: 262-279,1974$.

[21] E Tardos and K.D. Wayne. Simple generalized maximum flow algorithms. In Proceedings of the 6th International IPCO Conference on Integer Programming and Conmbinatorial Optimization, pages 310-324. Springer, 1998.

[22] H. Topaloglu and W.B. Powell. Sensitivity analysis of a dynamic fleet management model using approximate dynamic programming. Operations Research, 55(2):319-331, 2007.

[23] C.A. Tovey. A simplified NP-complete satisfiability problem. Discrete Applied Mathematics, 8:85-89, 1984.

[24] M.A. Turnquist and B.P. Markowicz. An interactive microcomputer-based model for railroad car distribution, 1989. Working Paper, Cornell University, Ithaca, NY.

[25] W. W. White and A. M. Bomberault. A network algorithm for empty freight car allocation. IBM Syst. J., 8:147-169, 1969. 